\title{
EQUATION OF STATE FOR HADRONIC MATTER
}

\author{
F. KARSCH and David E. MILLER ${ }^{1}$ \\ Department of Theoretical Physics, University of Bielefeld, Fed. Rep. Germany
}

Received 3 July 1978

An exact equation of state for a relativistic quantum gas model of hadronic matter is derived using analytical methods. Our results are shown to be an extension of the quantum gases through the inclusion of the hadronic mass spectrum $\rho(m)$.

The statistical description of a system of stronginteracting particles results in regarding the formed resonances as particles in a way similar to the statistical bootstrap model $[1,2]$ and to the dual resonance model $[3,4]$ with the mass spectrum in the large $m$ limit of the type

$\rho(m)=c m^{a} \exp \{b m\}$,

where $a, b, c$ are constants. The thermodynamical properties of hadronic matter are basically developed out of the theory of interacting quantum gases [5], where the interactions are replaced by an ideal gas containing the basic hadrons of mass $m_{0}$ and their resonances or intermediate states [6] expressed through $\rho(m)$. The structure of the ideal relativistic quantum gases [7] in invariant phase space [8] may be described in the grand canonical ensemble by the grand partition function $Z(\beta, V, A)$ where $\beta$ is the inverse temperature, $V$ the spatial volume and $A$ is the relativistic fugacity. The properties of $A$ in a relativistic Bose-gas model have been investigated [9] and extended to a system with a mass spectrum [10].

In the present work we shall rewrite $Z(\beta, V, A)$ for a simple relativistic Bose-gas model in invariant phase space with a mass spectrum in the form

$$
\begin{gathered}
Z(\beta, V, A)=\exp \left\{-\frac{V}{2 \pi^{2}} \int_{1}^{\infty} \mathrm{d} t t \sqrt{t^{2}-1}\right. \\
\left.\times \int_{m_{0}}^{\infty} \mathrm{d} m m^{3} \rho(m) \ln \left(1-A \mathrm{e}^{-m \beta t}\right)\right\} .
\end{gathered}
$$

\footnotetext{
${ }^{1}$ Heinrich-Hertz Fellow 1977-78.
}

From this equation we are immediately able to get the thermodynamical functions, in particular, we shall consider the pressure $p$ and the particle density $n$ in the thermodynamic limit as

$$
\begin{aligned}
& p(\beta, A)=\lim _{V \rightarrow \infty}\left[\frac{1}{V \beta} \ln Z(\beta, V, A)\right], \\
& n(\beta, A)=\lim _{V \rightarrow \infty}\left[\frac{1}{V} A \frac{\partial}{\partial A} \ln Z(\beta, V, A)\right] .
\end{aligned}
$$

By using the results of (2) we evaluate these thermodynamical quantities as

$$
\begin{aligned}
& p=\frac{A}{6 \pi^{2}} \int_{1}^{\infty} \mathrm{d} t \int_{m_{0}}^{\infty} \mathrm{d} m\left(t^{2}-1\right)^{3 / 2} \frac{m^{4} \rho(m)}{\exp \{m \beta t\}-A}, \\
& n=\frac{A}{2 \pi^{2}} \int_{1}^{\infty} \mathrm{d} t \int_{m_{0}}^{\infty} \mathrm{d} m t\left(t^{2}-1\right)^{1 / 2} \frac{m^{3} \rho(m)}{\exp \{m \beta t\}-A} .
\end{aligned}
$$

In the following discussion we shall show how it is possible to reduce the problem of the inversion of these equations of the solution of a well-known problem in singular integral equations the Hilbert problem [11]. This method was first applied by Leonard [12] in order to solve for the fugacity $A$ of the ideal quantum gases. Not long thereafter it was extended by Nieto [13] to find the fugacity and therefrom the equation of state for the ideal relativistic quantum gases. Although in the following work we shall only carry out the explicit derivation of the equation of state for a hadronic Bose system in the thermodynamic limit, there is no further mathematical difficulty in working 
out the same procedure for the hadronic Fermi system. However, the hadronic Fermi system presents difficulties in the determination of the mass spectrum. Furthermore, it is possible to include for the Bose gas the additional expressions arising from the zero momentum term relating to the Bose-Einstein condensation. We also remark that another approach to the equation of state of a hadronic system in a "correlation" volume has been discussed by Fré and Page [15] in the investigation using the statistical bootstrap equation with $A=1$.

Now we reformulate the expressions $(4 a, b)$ for the solution of $A$ using the formulation of the Hilbert problem [11]. First we define the transformation to the new variables as $x=\exp \{m \beta t\}$ and $y=t$, for which we write $(4 a, b)$ as

$p=F_{p}(A) / 6 \pi^{2} \beta, \quad n=F_{n}(A) / 2 \pi^{2}$,

where the functions $F_{\alpha}(a)$ with $\alpha$ replacing $p$ or $n$ are given by the integrals

$F(A)=A \int_{A^{*}}^{\infty} \frac{\mathrm{d} x f_{\alpha}(x)}{x(x-A)}$,

with $A^{*}=\exp \left\{m_{0} \beta\right\}$, the lower limit. The explicit forms of $f_{\alpha}(x)$ are given as

$f_{p}(x)=\int_{1}^{b(x)} \mathrm{d} y\left(t^{2}-1\right)^{3 / 2} m^{4} \rho(m) / y$,

$f_{n}(x)=\int_{1}^{b(x)} \mathrm{d} y t\left(t^{2}-1\right)^{1 / 2} m^{3} \rho(m) / y$,

where the upper bound of integration $b(x)$ is written as $(\ln x) / m_{0} \beta$ and the function $m(x, y)$ is given by $(\ln x) / \beta y$. Following Leonard [12] we define the function $P(x)$ of the complex variable $s$ as

$P(s)=1-F_{p}(s) / 6 \pi^{2} \beta p$,

which has a branch cut on the real axis $\left[A^{*}, \infty\right)$ and a zero at $s=A$. From the structure of the Hilbert problem we use the Plemelj formula [11] to find the limiting values of $P(s)$ above and below the branch cut as

$P^{ \pm}(s)=1-\operatorname{Pr}\left\{F_{p}(s) / 6 \pi^{2} \beta p\right\} \mp \mathrm{i} \pi f_{p}(s) / 6 \pi^{2} \beta p$,

where "Pr" refers to the principle value. From this formula we find that the ratio $P^{+}(s) / P^{-}(s)$ in the inter- val $\left[A^{*}, \infty\right)$ is expressed by $\exp \left\{2 \mathrm{i} \Theta_{p}(s)\right\}$ where the angle is found to be

$\Theta_{p}(s)=\arctan \left\{\pi f_{p}(s) /\left(F_{p}(s)-6 \pi^{2} \beta p\right)\right\}$.

The essential structure of the Hilbert problem is to find this function $P$ in terms of $P^{+} / P^{-}$by knowing only the limiting values on the branch cut. This mathematical problem may he uniquely solved as soon as the behaviour of $\Theta_{p}(s)$ on the cut together with all the singularities of $P(s)$ are known.

The examination of the asymptotic behaviour of the functions $f_{p}$ and $F_{p}$ shows that

$\lim _{s \rightarrow A^{*}} \tan \Theta_{p}(s)=0$,

$\lim _{s \rightarrow \infty} \tan \Theta_{p}(s)=(\pi b / \beta)(1-b / \beta)$.

The equation (11a) is identical to that found by Nieto [13] for the relativistic gas. However, the limit in (11b) differs from his for finite $\beta$ and $b$ when $\beta \neq b$. In our case we find for the chosen branch of the arctangent the following limiling conditions on the angles:

$\lim _{s \rightarrow A^{*}} \Theta_{p}(s)=-\pi$,

$\lim _{s \rightarrow \infty} \Theta_{p}(s)=h(b / \beta)$,

when $-\pi / 2<h(b / \beta)<0$ and $\lim _{\beta \rightarrow b} h(b / \beta)=0$.

Furthermore, we have the conditions that $P(A)$ is zero and that $P(s)$ diverges for large $s$. Because $A$ is the only singular point of $P(s)$, we find accordingly the welldefined solution of the Hilbert problem as

$P(s)=C \frac{s-A}{s-A^{*}} \exp \{\Gamma(s)\}$,

$\Gamma(s)=\frac{1}{\pi} \int_{A^{*}}^{\infty} \mathrm{d} x \frac{\Theta_{p}(x)}{x-s}$.

The equality of the two forms of $P(s)$ in the equations (8) and (13a) enables us to determine the constant $C$ together with $A$ through the examination of $P(s)$ and its first derivative at $s=0$. This solution yields as a result for $A$ in the form

$\frac{1}{6 \pi^{2} \beta p} \int_{A^{*}}^{\infty} \mathrm{d} x \frac{f_{p}(x)}{x^{2}}=\frac{1}{A}-\frac{1}{A^{*}}-\frac{1}{\pi} \int_{A^{*}}^{\infty} \mathrm{d} x \Theta_{p}(x) / x^{2}$.

The substitution of (7a), after we have taken the inverse 
of our above variable transformations, into the left hand side of (14) gives us the solution for $A$ as

$$
\begin{aligned}
\frac{1}{A} & =\frac{1}{A^{*}}+\frac{1}{\pi} \int_{A^{*}}^{\infty} \mathrm{d} x \frac{\Theta_{p}(x)}{x^{2}} \\
& +\frac{1}{2 \pi^{2} \beta^{2} p} \int_{m_{0}}^{\infty} \mathrm{d} m m^{2} \rho(m) K_{2}(\beta m),
\end{aligned}
$$

for which we have used the integral representation [14] for the modified Bessel functions of the second kind $K_{\nu}(x)$.

Similarly we may repeat the entire calculation for the particle density $n$ to find another solution for $A$ as

$$
\begin{aligned}
\frac{1}{A}= & \frac{1}{A^{*}}+\frac{1}{\pi} \cdot \int_{A^{*}}^{\infty} \mathrm{d} x \frac{\Theta_{n}(x)}{x^{2}} \\
& +\frac{1}{2 \pi^{2} n \beta} \int_{m_{0}}^{\infty} \mathrm{d} m m^{2} \rho(m) K_{2}(m \beta),
\end{aligned}
$$

whereby $\Theta_{n}(x)$ is defined analogously with (10).

For the equation of state we bring together (15) and (16) while solving for $1 / A$ so that,

$$
\begin{aligned}
& \left(\frac{1}{\beta p}-\frac{1}{n}\right) \int_{m_{0}}^{\infty} \mathrm{d} m m^{2} \rho(m) K_{2}(m \beta) \\
& \quad=2 \pi \beta \int_{A^{*}}^{\infty} \mathrm{d} x\left(\Theta_{n}(x)-\Theta_{p}(x)\right) / x^{2} .
\end{aligned}
$$

We can immediately see that the absence of the right hand side of (17) gives back the usual classical ideal gas equation of state. Thus the integral over these angles, when finite, expresses both the non-ideal cha. racter of the relativistic quantum gases and the properties of the hadronic system through $\rho(m)$ as seen from $(7 \mathrm{a}, \mathrm{b}),(10)$ and $(11 \mathrm{~b})$.

The specific nature of Bose effects have been studied previously by Engels et al. [16] for the statistical bootstrap model where their relative importance as well as the solution of this generalized model has been discussed. The effect of Bose-Einstein clustering [9] in hadronic matter has been considered. For the case $a=-3$ Bose - Einstein condensation has been found to take place [17] at a temperature under the ultimate (Hagedorn) temperature.

Furthermore, we notice from our equation of state (17) that in the limit $\beta \rightarrow b$ the properties vary depending upon $a$. For $a>-5 / 2$ we have from the divergence of the mass spectrum integral in equation (17) simply the ideal gas solution. At $a=-5 / 2$ we have the limiting case of a logarithmic divergence. The interesting case for $a<-5 / 2$ has the mass spectrum integral finite making the correction on the right hand side of (17) important. In all cases the limit of high temperature and low density gives back the basic ideal gas structure. Howcver, it is clcar that for the explicit examination of the Bose-Einstein condensation we must include the zero momentum term when $a<-5 / 2$.

We want to thank H. Satz and R. Hagedorn for discussions and for criticism of the manuscript. One of us (D.E.M.) would like to acknowledge the financial support of the Heinrich-Hertz-Stiftung.

\section{References}

[1] R. Hagedorn, Nuovo Cimento Suppl. 3 (1965) 147.

[2] S. Frautschi, Phys. Rev. D3 (1971) 2821.

[3] S. Fubini and G. Veneziano, Nuovo Cimento 64A (1969) 811.

[4] P. Frampton, Dual resonance model (Benjamin, New York, 1974).

[5] G.E. Uhlenbeck and E. Bethe, Physica 3 (1936) 729; 4 (1937) 915.

[6] S.Z. Belenkij, Nucl. Phys. 2 (1956) 259.

[7] M. Chaichian, R. Hagedorn and M. Hayashi, Nucl. Phys. B92 (1975) 445.

[8] B. Touscheck, Nuovo Cimento 58B (1968) 295.

[9] I. Montvay and H. Satz, Nuovo Cimento 39A (1977) 425.

[10] J. Letessier, A. Tounsi and H. Satz, Statistical bootstrap and pion condensation, Paris preprint, IPNO/TH 77/16r; Nuovo Cimento, to be published.

[11] N.I. Muskhelishvili, Singular integral equations (Noordhoff, Groningen, 1953).

[12] A. Leonard, Phys. Rev. 175 (1968) 221.

[13] M.M. Nieto, Lett. Nuovo Cimento 1 (1969) 677; J. Math. Phys. 11 (1970) 1346.

[14] G.N. Watson, Bessel functions (Cambridge, 1966).

[15] P. Fré and R. Page, Nuovo Cimento 35A (1977) 481.

[16] J. Engels, K. Fabricius and K. Schilling, Phys. Lett. 59B (1975) 477; Phys. Rev. D16 (1977) 189.

[17] R. Fiore, R. Page and L. Sertorio, Nuovo Cimento 44A (1978) 531. 